Running title: Geoffroy's cat ecology in Brazil

\title{
Density and spatio-temporal behaviour of Geoffroy's cats in a human-dominated
}

\section{landscape of southern Brazil}

Flávia P. Tirelli ${ }^{\mathrm{a}, \mathrm{b}, \mathrm{c}, \mathrm{d} *}$, Fábio D. Mazim ${ }^{\mathrm{d}, \mathrm{e}}$, Peter G. Crawshaw Jr. ${ }^{\mathrm{f}}$, Ana Paula Albano ${ }^{\mathrm{g}}$, Caroline Espinosa ${ }^{\mathrm{c}}$, Diego Queirolo ${ }^{\mathrm{h}}$, Fabiana Lopes Rocha ${ }^{\mathrm{i}}$, José Bonifácio Soares ${ }^{\mathrm{e}}$, Tatiane C. Trigo ${ }^{\mathrm{j}}$, David W. Macdonald ${ }^{\mathrm{b}}$, Mauro Lucherini ${ }^{\mathrm{k}}$, and Eduardo Eizirik ${ }^{\mathrm{a}, \mathrm{d}}$

${ }^{a}$ PUCRS, Escola de Ciências, Laboratório de Biologia Genômica e Molecular, 6681 Ipiranga Av., Porto Alegre, RS, 90619-900, Brasil

${ }^{b}$ Wildlife Conservation Research Unit (WildCRU), Department of Zoology, University of Oxford, The Recanati-Kaplan Centre, Tubney House, Tubney, Abingdon Road, OX13 5QL, United Kingdom

${ }^{\text {c} P r o g r a m a ~ d e ~ P o ́ s-g r a d u a c ̧ a ̃ o ~ e m ~ B i o l o g i a ~ A n i m a l, ~ I n s t i t u t o ~ d e ~ B i o c i e ̂ n c i a s, ~ U n i v e r s i d a d e ~}$ Federal do Rio Grande do Sul (UFRGS), Av. Bento Gonçalves 9500, prédio 43435, Bairro Agronomia, Porto Alegre, RS, 91501-970, Brasil

${ }^{\mathrm{d}}$ Instituto Pró-Carnívoros, Atibaia, SP, Brazil

${ }^{\mathrm{e}}$ Ka'aguy Consultoria Ambiental, Av. Adolfo Fetter 1092, B, Laranjal. Pelotas, RS, 96083-000, Brasil

${ }^{\mathrm{f}}$ Centro Nacional de Pesquisa e Conservação de Mamíferos Carnívoros (CENAP-ICMBio), Estrada Hisaichi Takebayashi, 8600, Usina Atibaia, SP, 12952-011, Brasil

${ }^{g}$ Hospital de Clínica Veterinária, Universidade Federal de Pelotas (UFPel), Avenida Eliseu Maciel, Jardim América. Capão do Leão, RS, 96010-610, Brasil

${ }^{\text {h}}$ Centro Universitario Regional Noreste, Universidad de la República, Ituzaingó 667, Rivera, 40000, Uruguay

${ }^{\mathrm{i}}$ Programa de Pós-graduação em Ecologia e Monitoramento Ambiental, Universidade Federal da Paraíba, Campus IV, Estr. Engenho Novo, s/n, Mamanguape, PB, 58280-000, Brasil

${ }^{j}$ Setor de Mastozoologia, Fundação Zoobotânica do Rio Grande do Sul (FZB), Rua Dr. Salvador França, 1427, Porto Alegre, RS, 90690-000, Brasil

${ }^{\mathrm{k}}$ Grupo de Ecología Comportamental de Mamíferos (GECM), Cátedra de Fisiología Animal, Departamento de Biología, Bioquímica y Farmacia, Universidad Nacional del Sur - CONICET, San Juan 670, 8000 Bahía Blanca, Argentina

*Corresponding author: Flávia P. Tirelli, Programa de Pós-graduação em Biologia Animal, Instituto de Biociências, Universidade Federal do Rio Grande do Sul (UFRGS), Av. Bento Gonçalves 9500, prédio 43435, Bairro Agronomia, Porto Alegre, RS, 91501-970, Brasil flavia.tirelli@gmail.com 
Abstract

Geoffroy's cat is a small Neotropical felid, seemingly abundant throughout most of its range and exhibiting considerable ecological plasticity. In Brazil, the species is restricted to the Pampas, one of the most threatened biomes in the country, where information on its ecology is scarce. Here we report the first assessments of its density, habitat selection, and activity patterns in Brazil. The study was conducted in humandominated landscape at the extreme south of the country. Using Spatially Explicit Capture-Recapture (SECR) analyses, the estimated population density ranged from $34.54( \pm 13.51 \mathrm{SE})$, based on camera trap and radio-telemetry data, to $41.78( \pm 16.12 \mathrm{SE})$ individuals $/ 100 \mathrm{~km}^{2}$, based only on camera trap data. A Resource Selection Function (RSF) analysis showed that our study population selected sites with native forest and rivers, and avoided areas close to roads, which probably implies avoidance of human contact. Although we observed a slight increase in its nocturnal activity during springsummer with respect to fall-winter, this population was predominantly nocturnal throughout the year. The use of grasslands and croplands (non-protected areas) was non-uniformly distributed through the hours of the day, whereas native forest was used more uniformly.

Key words: Activity patterns; Brazilian Pampas; Habitat use; Leopardus geoffroyi; Ranchlands.

\section{Introduction}

Behavioural and ecological features of a species influence its response to human

disturbance (Frid and Dill, 2002; Ciuti et al., 2012). While some species are driven to

extinction in human-dominated landscapes, others persist, raising the question of what

biological features underlie such discrepant outcomes. Geoffroy's cat (Leopardus

geoffroyi) is a small, solitary Neotropical felid categorized by the IUCN as "Least

Concern" (Pereira et al., 2015). This cat is considered abundant over most of its

distribution, ranging from Bolivia and southernmost Brazil to the southern tip of South

America (Macdonald and Loveridge, 2010; Cuyckens et al., 2016). Studies in

Argentina, the country occupying the largest proportion of its distribution, indicate that

Geoffroy's cat is tolerant of (and perhaps sometimes favoured by) habitat alteration and can persist alongside livestock ranching and agriculture. This has led to the suggestion 
that Geoffroy's cat is an ecologically adaptable species (Castillo et al., 2008; Pereira et al., 2012; Pereira et al., 2015; Caruso et al., 2016). Testing this hypothesis using multiple (and standardized) approaches, and at different field sites, is therefore important to improve understanding of this species' ecology and its resilience to humaninduced disturbances.

Studies carried out in Bolivia, Argentina and Chile estimated very different population densities at sites with contrasting ecological features (W. Johnson, personal communication, in Nowell and Jackson, 1996; Cuellar et al., 2006; Pereira et al., 2011;

Caruso et al., 2012). Although some of these differences might be attributable to different methodologies (such as different size of sample areas in capture-recapture analysis), much of the intra-specific variation appears to reflect adaptation to contrasting environmental factors, including human disturbance. There are no estimates of population density of Geoffroy's cat throughout large areas of Paraguay, Uruguay and Brazil, although it is usually considered abundant in Uruguay and Brazil (Soutullo et al., 2010; T. Trigo, personal observation 2014, in Pereira et al., 2015). Insofar as most previous studies of Geoffroy's cat population density used traditional capture-recapture models, improved estimates can now be made using spatially-explicit capture-recapture analysis (SECR: Royle et al., 2013; Efford, 2018). SECR models incorporate the spatial information of captures, and resolve the "unknown sample area" problem. Geoffroy's cats mostly occur in open landscapes. However, within these landscapes, they can use open as well as closed habitats (Johnson and Franklin, 1991; Manfredi et al., 2006; 2012; Pereira et al., 2006; Pereira et al., 2012, Caruso et al., 2016; Castillo et al., 2019). Regarding activity patterns, this felid seems to be more crepuscular and nocturnal than diurnal (Johnson and Franklin, 1991; Cuellar et al., 2006; Manfredi et al., 2011; Pereira et al., 2011), although one report documents a 
switch to diurnality in response to food shortage (Pereira, 2010). Taken together, these observations suggest that Geoffroy's cat is flexible, and adaptable to diverse environments, although many aspects of its ecology remain poorly known throughout most of its range. To improve this knowledge and specifically to define the limits of its intra-specific ecological variation, it is interesting to investigate in detail how Geoffroy's cat behaves at the extremes of its geographical range. The Brazilian Pampas is the only biome in Brazil where Geoffroy's cat occurs (Pereira et al., 2015; Cuyckens et al., 2016), and has been categorized as a region with medium-to-high predicted habitat suitability for this species (Cuyckens et al., 2016). This region represents the north-eastern extreme of its range, and is thus an interesting target for ecological research, but so far very few studies have investigated this species in this area (Sousa and Bager, 2008; Trigo et al., 2013; Tirelli et al., 2018). In this context, we aimed to estimate population density and to characterize behavioural patterns of Geoffroy's cats in a human-dominated landscape typical of this region. More broadly, we aimed to identify the factors underlying its apparent resilience (and potential limits of that resilience) to anthropogenic habitat alterations.

2

\section{Material and methods}

\section{Study area}

The study was conducted from November 2013 to September 2015 in two contiguous, private properties located in the Pampas ecosystem of southernmost Brazil $\left(30^{\circ} 04^{\prime} \mathrm{S}, 55^{\circ} 31^{\prime} \mathrm{W}\right)$. The study area covered $\sim 30 \mathrm{~km}^{2}$, and consisted of a mosaic of natural habitat remnants, cattle pastures and croplands in the surroundings of a stream 
131 ('Arroio Caverá') (Fig. 1). The native vegetation is characterized by a diversity of

grasslands (e.g., Aristida murina on shallow soils and Paspalum notatum on deeper soils) (Boldrini, 2009) and by riparian forests adjacent to the stream, represented mainly by Sebastiania schottiana, Phyllantus sellowianus, Pouteria salicifolia and Syagrus romanzoffiana (Guadagnin et al., 2015). All riparian vegetation is included in legal reserve and permanent preservation areas, which are areas instituted by the Brazilian Forest code (Brazilian law 12.651/2012) determining the minimal percentage of native vegetation that needs to be maintained in rural properties. These areas should be kept free from cattle, but are sometimes impacted by incursions of livestock attempting to access water. Cattle grazing is conducted on native grasses, and livestock management practices are typical for the region, with $\sim 90$ cattle $/ \mathrm{km}^{2}$, and husbandry activity (using herding dogs) occurring during daytime, every day of the year (F. Tirelli, personal observation, 2013-2015). Crops cover $c a$. 40\% of the proprieties and comprise soybean, rice, oat, and ryegrass. The annual rainfall averages $1500 \mathrm{~mm}$, and the mean temperature is $13^{\circ} \mathrm{C}$ in winter and $24^{\circ} \mathrm{C}$ in summer (Nimer, 1989).

\section{Camera Trapping}

Camera trap data were collected from November 2013 to September 2015, with a gap in sampling between March and May 2014, as reported by Tirelli et al. (2018). We employed 26 digital camera traps (20 Scout Guard ${ }^{\circledR}, 4$ Bushnell $^{\circledR}$ and 2 Moultrie $^{\circledR}$ ), which were positioned $c a .30 \mathrm{~cm}$ above the ground, with no lure. Cameras were active 24 hours per day on video mode (20 seconds), and were checked weekly to ensure functionality. The total sampling effort was estimated by multiplying the number of survey days by the number of functional camera traps per day. 

activity (tracks, faeces or radio-telemetry data). We employed two different camera trap sampling protocols: (i) a camera trap layout aiming to collect data for density analysis, used from January to August 2015 (we used two surveys of 48 days each to analyse density), consisting of 13 stations, either inside or immediately outside the riparian vegetation (Fig. 1), with paired cameras (to record both sides of an individual); and (ii) for the remaining time of the study (2013-2014 and September 2015) cameras were placed either individually or in pairs, covering a broader portion of the study area and specifically aiming to collect data for the analysis of activity patterns. In every case, the spacing between stations was maintained at ca. $600 \mathrm{~m}$, the radius of the smallest home range recorded for the species (Pereira et al., 2012).

Live capture and radio telemetry monitoring out at all times under the supervision of a veterinarian (see Tirelli et al. [2018] for details on capture and radio-telemetry procedures). The study was also approved by the university animal care and use ethics committee (PUCRS CEUA - 14/00400), and followed the recommendations of the guidelines of the American Society of with activity and mortality sensors (Telonics; MOD-080-2) between May 2014 and to September 2015 at different times of the day, aiming to obtain at least three data 
points (including location and activity) per individual in each 1-hr period within the 24hour cycle. For each data point, the spatial location of the individual was estimated by triangulation, using at least three different bearings (taken at most 10 min apart) per point. More information on this aspect of the methodology can be found in Tirelli et al. (2018). Activity readings relied on the activity sensor built into each radio-collar, which differentiated active ( $>43$ beats per minute $[\mathrm{BPM}])$ from non-active $(\leq 43 \mathrm{BPM})$ states.

The threshold between the two states was defined empirically by cross-validation between the activity reading and direct observation of individuals during this study. We recorded the BPM of each individual every time a location bearing was collected, and then calculated the average activity (in BPM) for that particular data point. This average activity value indicated if the cat was active or non-active.

\section{Density estimates}

Two standardized survey periods were delimited from within our continuous camera trapping sampling to estimate population density at our study site. One of them targeted the summer (January and February 2015) and the other the winter (end of June to the beginning of August 2015). Both periods were limited to 48 days to meet the assumption of population closure (Balme et al., 2009). For this analysis, each camera trap record was examined carefully and only those for which it was possible to discern the spotting pattern on both flanks or on other body parts (e.g. forehead markings or tail stripes) (Pereira et al., 2011) were retained in the dataset. These patterns were then drawn, producing a reference database that was used for individual identification. To maintain temporal independence between individual captures, each individual was counted only once per camera station in a 24-hour period. 
Density was estimated with SECR models (Royle et al., 2013), using the package 'secr' (Efford, 2018) in R-3.2.3 (R Development Core Team, 2015). Because this approach allows density to be estimated by combining different types of data (Efford, 2018), we complemented our camera trapping records with animal locations obtained by radio telemetry. We only included the telemetry data from individuals that were monitored during the survey periods delimited for camera-trap sampling. Since capture probabilities may be influenced by sex, age, and particular behavioural features, we built six different models including combinations of these variables to represent capture history, and tested their relative performances using the Akaike Information Criterion (AIC) (Akaike, 1974). In addition, to allow direct comparisons between our density estimates and others reported previously (Cuellar et al., 2006; Pereira et al., 2011; Caruso et al., 2012), we also estimated population density using telemetry data only (Benzaquín, 2008; Luengos Vidal et al., 2012), employing traditional CaptureRecapture methods using MMDMOSA, MMDM, HMMDM, and home range (HR) radius to estimate the size of the sampled area (Supplementary material S1).

Resource selection functions (RSF) distance to roads and distance to water (Supplementary material S6). We chose these variables because the mosaic of habitat composition and roads are the main features of the area, and water is often a crucial resource. To categorize habitat, we downloaded a Landsat-7TM satellite image of the study site from the National Institute of Space Research, Brazil (INPE), and used ArcGIS-10.4.1 (ESRI, 2016) to generate a supervised classification of its habitats using a maximum likelihood approach (Patil et 
al., 2012). We used the following three categories: native forest (NF), grassland (GS), and cropland (CR). As for the other two variables, we downloaded shape files of the road network and rivers from the Rio Grande do Sul (RS) state "Fundação Estadual de Proteção Ambiental Henrique Luiz Roessler” (FEPAM). Subsequently, we used the Euclidian distance tool in ArcGIS-10.4.1 to generate the maps of distances (Supplementary material S6b and c).

The analyses of animal resource selection functions (RSF) were calculated based on the locations of individuals obtained via telemetry. We computed the selection ratios for $3^{\text {rd }}$ order selection (Johnson, 1980). We used only this order because, according to Walter and Fischer (2016), the use of the $2^{\text {nd }}$ order selection in resource selection analysis is necessary only across distinct landscapes. We calculated the extent of area occupied by using a Minimum Convex Polygon 100\% (MCP) for each individual using the package 'adehabitatHR' (Calenge, 2006) in R software. Within each Geoffroy's cat MCP, we compared the values of variables extracted from the locations of each animal (resources used) with those of a random sample of 500 points (resources available) (Walter and Fischer, 2016). We then used General Linear Models (GLM) by employing the function glmer of the package 'Ime4'1.1-17 (Bates et al., 2015) in R software. We created nine distinct models including different combinations of the variables to characterize the use of resources, and compared the models using the AIC. Finally, from our top model, we created a map of predicted habitat suitability using 'adehabitatHS', according to Walter and Fischer (2016). 
We investigated activity patterns using both camera trap and telemetry data. We only included images obtained at least one hour apart from each other, which were then assumed to represent independent events. We estimated activity patterns for each 1-hr partition of the 24-hour cycle of the day, considering both the total survey period and two separate seasonal partitions: spring-summer (October to March) and fall-winter (April to September). These partitions were defined based on their distinct patterns of sunrise and sunset at the study site during the survey period (Supplementary material S5).

We used two analytical approaches for both the total survey period and seasonal partitions: the Ridout and Linkie (2009) methods and Rayleigh's Uniformity test (Fisher, 1993). We initially used the Ridout and Linkie (2009) methods to fit Von Mises kernel density functions to times of observations of Geoffroy's cats in the total survey period and in the seasonal partitions, and to estimate the coefficient of overlap $\left(\Delta_{4}\right)$ between the two seasons (the coefficient ranges from 0 [no overlap in activity patterns] to 1 [complete overlap]). These analyses were performed using the package 'overlap' (Meredith and Ridout, 2014) in R-3.2.3. In addition, we used Rayleigh's Uniformity test (implemented in the software Oriana-4.02 [Kovach, 2013]) to calculate the probability of the null hypothesis that the data were distributed uniformly, for both the total survey period and the seasons.

For the telemetry-based analyses, we considered the proportion of activity or inactivity, which was obtained by dividing the number of 'active' or 'inactive' data points by the total number of data points in each 1-h period. In this case, we only used Rayleigh's Uniformity test, since the Ridout and Linkie (2009) methods are not applicable to the proportional approach employed here. 

the hours of the day in the different habitat types (NF, GS, CR), we used Rao's Spacing Test (Kovach, 2013), and to test if activity patterns were significantly different among habitats, we employed Watson's $U^{2}$ test (Kovach, 2013). For these analyses, we used the software Oriana-4.02 and only telemetry data because our camera trap layout did not sample the different habitat types equally.

Results

The overall camera trap sampling effort in this study was 8,845 trap-days, resulting in 522 felid images. Of these, 516 (98.85\%) were Geoffroy’s cats, two $(0.38 \%)$ were margays (Leopardus wiedii), and four $(0.77 \%)$ were feral domestic cats (Felis catus). In addition, 12 Geoffroy's cat individuals were live-captured, seven of which (four males and three females) were radio-collared and monitored (Supplementary material S2). The five additional individuals that were not monitored included one cat that was killed by domestic dogs, one failed collar, and three individuals that were captured in the last recapture campaign. Additionally, one of female Geoffroy's cat died two months after its capture. We suspect that this female was poisoned, because when we carried out a field necropsy, we found that its internal organs were melted. 
The SECR density estimate was performed using two data sets ('camera trap

only' and 'combined', which used both camera trap and telemetry data) for two different surveys (summer and winter). Each survey comprised 624 camera trap-days. In the summer survey, we identified 11 different Geoffroy's cat individuals (four males and seven females) in 49 independent camera trap captures. For five of them (three males and two females), we obtained telemetry data (37 total points) that were incorporated in the combined density estimates. In the winter survey, we recognized 11 distinct individuals (five males and six females) in 33 independent camera trap captures; 9 individuals (three males and six females) overlapped with those identified in the summer. In this case, we could only include telemetry data from two females (17 total points), which likely biased the combined density estimates for this season. The trap-specific model (bk) (Table 1) provided the best fit for both types of data in both surveys. The $\Delta \mathrm{AIC}$ was $>2$ for the second most likely models in the majority of cases, except for 'Winter combined' (Supplementary material S2). The density estimates based on the SECR approach ranged from 34.5 to 41.78 individuals $/ 100 \mathrm{~km}^{2}$, and were extremely similar for the two seasons when using camera trap data only (Table 1).

In addition to the SECR approach, we also estimated density using telemetry data only, as well as traditional capture-recapture methods based on camera-trap or combined data (see Supplementary material S1 for details). Most of these estimates overlapped with those obtained with the SECR method, and provided a framework for direct comparisons with results from previous studies (Table 2).

\section{Resource selection}


The RSFs were estimated using 434 telemetry locations from seven Geoffroy's cats (four males and three females) (Supplementary aterial S3). The model with the best AIC value included all three variables (native forest, distance to roads, and distance to water [Table 3; Supplementary material S4]). Native forest and distance to roads influenced the habitat use of Geoffroy's cats positively, whereas distance to water had a negative effect (Table 3). The best model generated a map with greater habitat suitability for areas in proximity to native forest and water (Fig. 2).

\section{Activity patterns}

The camera trap data set used to estimate activity patterns comprised 513 records. The data for the complete survey indicated that Geoffroy's cats were mostly active during the night, with $71.6 \%$ of active observations between sunset and sunrise (19:04 and 07:02, respectively) (Fig. 3). When the seasons were assessed separately (240 records in summer-spring and 273 in fall-winter), cats were mostly active during the night in summer-spring ( $78.8 \%$ of observations) and during nocturnal/crepuscular hours in fall-winter (72.5\% of observations) (Fig. 4). The intensity of activity decreased before sunrise and increased before sunset in both seasons, although in summer the nocturnal peak of activity was slightly higher than in winter. The coefficient of overlap in activity patterns between seasons was $\Delta_{4}=0.79$ (CI 0.7-0.85) (Fig. 4). The mean activity time (average $\pm \mathrm{SD}$ ) was 00:37 \pm 04:44 for spring-summer and 22:03 \pm 06:56 for fall-winter (Supplementary material S7). For all data sets, our results showed that the activity patterns were not uniformly distributed throughout the day (Rayleigh's Uniformity test: $\mathrm{Z}=20.97, \mathrm{P}<0.01$ for spring-summer; $\mathrm{Z}=3.72, \mathrm{P}=0.02$ for fall-winter). 
collared cats (the activity sensor of one of the collars failed). Since only the active fixes are informative for this analysis (which limited the available sample size), we differences in time-specific peaks are apparent between camera trapping and telemetry data, both data sets indicated a similar mean time of activity for the survey period (camera trapping: 23:36 \pm 06:04; radio-tracking: 23:19 \pm 03:47) and non-uniformity in the use of the 24-hour cycle ( $\mathrm{Z}=8.03, \mathrm{P}<0.01$ and $\mathrm{Z}=3.35, \mathrm{P}=0.03$, respectively; Fig. 5). GS $(18 \%)$. Mean activity time in NF was 21:00 \pm 02:16, while in GS was 04:00 \pm 5:39 and in CR was 22:00 \pm 01:39. Given the strong trend for predominantly nocturnal activity in all three habitat types (NF: 59\%; CR: 67.6\%; GS: 60\%), results of Watson's pairwise test (which tests whether activity patterns differed between habitats) were nonsignificant (NF vs. GS: $\mathrm{U}^{2}=0.08, \mathrm{P}>0.2 ; \mathrm{NF}$ vs. $\mathrm{CR}: \mathrm{U}^{2}=0.02, \mathrm{P}>0.5$; $\mathrm{CR}$ vs. GS: $\left.\mathrm{U}^{2}=0.06, \mathrm{P}>0.5\right)$. However, when each habitat type was assessed separately, contrasting patterns emerged, as the activity was significantly non-uniformly distributed throughout the hours of the day in GS (Rao's Spacing Test; $U=202.32, \mathrm{P}<0.01)$ and $\mathrm{CR}(\mathrm{U}=186.12$, $\mathrm{P}<0.05)$, whereas for $\mathrm{NF}$ the distribution was uniform $(\mathrm{U}=116.04, \mathrm{P}>0.5)$ (Supplementary material S8).

\section{Discussion}


378 the study area and in the nocturnal hours. Density estimations for Geoffroy's cat have

379 ranged from seven (W. Johnson, personal communication, in Nowell and Jackson

380 [1996]) to 290 (Pereira et al., 2011) individuals $/ 100 \mathrm{~km}^{2}$. Our results indicate that

381 Geoffroy's cat in the Brazilian Pampas exhibit medium-to-high density when compared

382 to previously assessed populations, in agreement with Cuyckens et al. (2016), who

383 predicted a medium-to-high suitability for the species in the region. Our density

estimates using SECR approaches were similar to those reported by Caruso et al.'s

(2012) for an area with a similar habitat in Argentina. The landscapes of both study

by cattle pastures and croplands. These alterations probably also influenced other

sympatric felid species. Only two of the seven felid species originally distributed in our study area (Espinosa et al., 2017; IUCN, 2019) were recorded, and the margay was very likely rare. A less diverse felid guild may reduce intraguild competition and release resources that Geoffroy's cat can exploit to reach increased population densities. This is in accordance with the hypothesis proposed by Cuyckens et al. (2016), that Geoffroy's cat is probably expanding its geographical distribution into human-modified land, and with the observation by Caruso et al. (2016) that this cat responded positively to the degree of disturbance and fragmentation, possibly favoured by limited human persecution.

However, there seems to be a limit to this adaptability (Castillo et al., 2008), since totally open areas such as dry grasslands appear to be unsuitable (Caruso et al., 2016; Manfredi et al., 2012). In this study, we observed that this Geoffroy's cat population did not use environmental resources uniformly, selecting areas that were and Fahrig (2012), with respect to some mammals being most vulnerable to the negative 
403 effects of roads, we suggest that the avoidance of areas close to roads by this Geoffroy's 404 cat population is related to human disturbance (including roads themselves and other 405 impacts that co-occur with them). The preference for covered habitats has been 406 described by other studies (Manfredi et al., 2006; 2012; Pereira et al., 2006; 2012;

407 Castillo et al., 2019), and is supported by our results. The significant and positive 408 relationship with water and native forest in the Brazilian pampas could be explained by the resources and shelter provided by these habitats in this non-protected area. In addition to the results on selectivity, we recorded videos with males and females urinating and sniffing in riparian vegetation, supporting the idea of Manfredi et al. (2006) and Soler et al. (2009), that wooded areas could also be used as 'communication centres' in open landscapes. Finally, in the periods when this nocturnal felid was active during the day the individuals mainly used native forest. This result supports the hypothesis that these areas provide food and valuable shelter during daylight, and indicate that legally preserved forested areas are essential for wildlife in this region, highlighting the importance of enforcing their protection across this landscape in southern Brazil.

In spite of some seasonal variation, possibly associated with fluctuations in prey availability and/or preference (Pereira, 2010), during both seasons Geoffroy's cats exhibited significantly nocturnal habits in our study area, as observed previously in the Humid Pampas (Manfredi et al., 2011; Castillo et al., 2008), Low Monte-Espinal 423 (Pereira et al., 2011), Dry Chaco (Cuellar et al., 2006), and Magellanic subpolar forest 424 (Johnson and Franklin, 1991). Different sources of data (camera trap and telemetry) consistently located the peak of activity around $23 \mathrm{~h}$, which supports the reliability of this result. In short, Geoffroy's cats are nocturnal and crepuscular, with a seasonal 427 tendency to cathermerality. 
avoidance of roads, and the essentially nocturnal habits (with diurnal activity limited to

430

431

432 native forest) in the Brazilian pampas are likely to be behavioural characteristics that enable this small cat to persist, with a reasonably high population density, in a humandominated landscape in which other felids have already declined or disappeared completely. Incorporating these findings into long-term conservation planning for this region (e.g. maintaining or increasing areas of native vegetation interspersed among human-altered habitats) should be relevant not only to ensure the persistence of Geoffroy's cat, but also to maintain or restore other components of its native biota.

\section{Acknowledgements}

We acknowledge Panthera, Fundação Grupo O Boticário/Brazil (1020_20142), and $\mathrm{CNPq} / \mathrm{Brazil}$ for funding this study. This study was also financed in part by the Coordenação de Aperfeiçoamento de Pessoal de Nível Superior - Brazil (CAPES) Finance Code 001. F.P.T. was supported by scholarships from CNPq and CAPES, and F.L.R. by PNPD/CAPES. We thank the ranch owners for authorizing us to conduct research on their lands. We are also grateful to CENAP/ICMBio/Brazil for lending camera-traps; and to C. B. Kasper, J. Pereira, N. Caruso, M. M. Guerisoli, and all others who assisted with fieldwork, especially P. O. Faria, B. Z .Silva, T. Michel, L. Teixeira and E. de Souza.

(1)

\section{References}


454 Akaike H., 1974. A new look at the statistical model identification. IEEE T. Automat.

455 Contr. 19(6), 716 - 723. https://doi.org/10.1109/TAC.1974.1100705

456 Benzaquín M., 2008. Área de acción del gato montés (Leopardus geoffroyi), en una 457 zona de la provincia fitogeográfica del Espinal. Chasicó, Pdo. de Villarino. Buenos

458 Aires. B.Sc. thesis, Departamento of Biology, Biochemistry and Pharmacy,

459 Universidad Nacional del Litoral, Santa Fé, Argentina.

460 Balme G. A., Hunter L. T. B., Slotow R., 2009. Evaluating methods for counting cryptic 461 carnivores. J. Wildlife Manage. 73, 433 - 441. https://doi.org/10.2193/2007-368

Bates D., Maechler M., Bolker B., Walker S., 2015. Fitting Linear Mixed-Effects Models Using lme4. J. Stat. Softw. 67(1), 1 - 48. https://doi.org/10.18637/jss.v067.i01

Boldrini, I. I., 2009. A Flora dos Campos do Rio Grande do Sul, in: Pillar, V. P., Müller, S.C., Castilhos, Z.M.S., Jacques, A.V.A. (Eds). Campos sulinos - conservação e uso sustentável da biodiversidade. MMA, Brasília, pp. 63-77

467 Calenge C., 2006. The package adehabitat for the R software: a tool for the analysis of space and habitat use by animals. Ecol. Model. 197, 516 - 519. https://doi.org/10.1016/j.ecolmodel.2006.03.017

470 Caruso N., Manfredi C., Luengos Vidal E. M., Casanave E. B., Lucherini M., 2012. 471 First density estimation of two sympatric small cats, Leopardus colocolo and Leopardus 472 geoffroyi, in a scrubland area of central Argentina. Annal. Zool. Fennici. 49, 18 - 191. 473 https://doi.org/10.5735/086.049.0306

474 Caruso N., Lucherini M., Fortin D., Casanave E. B., 2016. Species-Specific responses 475 of carnivores to human-induced landscape changes in Central Argentina. PLoS ONE 476 11(3), e0150488. https://doi.org/10.1371/journal.pone.0150488

477 Castillo D., Luengos Vidal, E., Caruso, N., Manfredi, C., Lucherini, M., Casanave E. 478 B., 2019. Spatial organization and habitat selection of the Geoffroy's cat in the Espinal 479 of central Argentina. Mamm. Biol. 94, 30 - 37.

480 https://doi.org/10.1016/j.mambio.2018.12.003

481 Castillo D., Luengos Vidal E., Lucherini M., Casanave E. B., 2008. First report on the 482 Geoffroy's cat in a highly modified rural area of the Argentine Pampas. Cat news. 49, $483 \quad 27-28$.

484 Ciuti S., Northrup J.M., Muhly T. B., Simi S., Musiani M., Pitt J., Boyce M., 2012. 485 Effects of humans on behaviour of wildlife exceed those of natural predators in a 486 landscape of fear. PLoS ONE. 7(11), e50611.

487 https://doi.org/10.1371/journal.pone.0050611 
Cuellar E., Maffei L., Arispe R., Noss A., 2006. Geoffroy's cat at the northern limit of their range: activity patterns and density estimates from camera trapping in Bolivian dry forest. Stud. Neotrop. Fauna Enrviron. 41, 169 - 177. https://doi.org/10.1080/01650520600840001

Cuyckens G. A. E., Pereira J. A., Trigo T. C., Da Silva M., Gonçalves L., Huaranca J. C., Bou N. P., Cartes J. L., Eizirik E., 2016., Refined assessment of the geographic distribution of Geoffroy's cat (Leopardus geoffroyi) (Mammalia: Felidae) in the Neotropics. J. Zool. 298, 285 - 292. https://doi.org/10.1111/jzo.12312

Efford M. G., 2018. secr: Spatially explicit capture-recapture models. R package version 3.1.6. Available from https://CRAN.R-project.org/package=secr (accessed 9 March 2018)

Environmental Systems Research Institute-ESRI., 2016. ArcGIS 10.4.1 desktop. Environmental Systems Research Institute, Redlands, CA.

Espinosa C. C., Trigo T.C., Tirelli F.P., da Silva L.G., Eizirik E., Queirolo D., et al., 2017. Geographic distribution modeling of the margay (Leopardus wiedii) and jaguarundi (Puma yagouaroundi): a comparative assessment. J. Mammal. 99, 252 262. https://doi.org/10.1093/jmammal/gyx152

Fisher N. I., 1993. Statistical analysis of circular data, Cambridge University Press Cambridge.

Frid A., Dill L. M., 2002. Human-caused disturbance stimuli as a form of predation risk. Conserv. Ecol. 6(1), 11. https://doi.org/10.5751/ES-00404-060111

Guadagnin, P. M. A., Trentin, R., da Silva Alves, F., 2015. Relação entre as variáveis Geomorfométricas e a Vegetação Florestal na Bacia Hidrográfica do Arroio CaveráOeste do RS. Rev. Dep. Geo. 29, 246-261. https://doi.org/10.11606/rdg.v29i0.102131

IUCN., 2019. The IUCN Red List of threatened species. Ver. 2019.1. Available from www.iucnredlist.org (accessed 10 January 2019).

Johnson D. H., 1980. The comparison of usage and availability measurements for evaluating resource preference. Ecology. 61(1), 65 - 71 .

\section{https://doi.org/10.2307/1937156}

Johnson W. E., Franklin W. L., 1991. Feeding and spatial ecology of Felis geoffroyi in Southern Patagonia. J. Mammal. 72(4), 815 - 820. https://doi.org/10.2307/1381848

Kovach W. L., 2013. Oriana 4.02 for Windows. Kovach Computing Services, Pentraeth.

Luengos Vidal E., Sillero-Zubiri C., Marino J., Casanave E., Lucherini M., 2012. Spatial organization of the Pampas fox in a grassland relict of central Argentina: a 
523 flexible system. J. Zool. 287, 133 - 141. https://doi.org/10.1111/j.1469-

$524 \quad$ 7998.2011.00896.x

525 Macdonald D.W., Loveridge A. J., 2010. Biology and conservation of wild felids.

526 Oxford University Press, Oxford.

527 Manfredi C., Soler L., Lucherini M., Casanave E. B., 2006. Home range and habitat use

528 by Geoffroy's cat (Oncifelis geoffroyi) in a wet grassland in Argentina. J. Zool. 268,

$529381-387 . \underline{\text { https://doi.org/10.1111/j.1469-7998.2005.00033.x }}$

530 Manfredi C., Lucherini M., Soler L., Baglioni J., Vidal E. L., Casanave E. B., 2011.

531 Activity and movement patterns of Geoffroy's cat in the grasslands of Argentina.

532 Mammal. Biol. 76(3), 313 - 319. https://doi.org/10.1016/j.mambio.2011.01.009

533 Manfredi C., Vidal E. L., Castillo D. F., Lucherini M., Casanave E. B., 2012. Home

534 range size and habitat selection of Geoffroyi's cat (Leopardus geoffroyi), Felidae,

535 Carnivora) in the pampas grassland.. 76 (1), $105-108$.

536 https://doi.org/10.1515/mammalia-2011-0039Mammalia

537 Meredith M. ,Ridout M., 2014. Overview of the overlap package. R. Proj., 1-9.

538 Nimer E., 1989. Climatologia do Brasil. Fundação IBGE, Rio de Janeiro.

539 Nowell K., Jackson P., 1996. Wild cats: Status Survey and Conservation Action Plan.

540 IUCN, Gland.

541 Patil M. B., Desai C. G., Umrikar B. N., 2012. Image classification tool for land

542 use/land cover analysis: A comparative study of maximum likelihood and minimum

543 distance method. Int. J. Geol. Earth Environ. Sci. 2(3), 189 - 196.

544 Pereira J. A., Fracassi N. G., Uhart M. M., 2006. Numerical and spatial responses of

545 Geoffroyi's cat (Oncifelis geoffroyi) to prey decline in Argentina. J. Mammal. 87, 1132

546 - 1139. https://doi.org/10.1644/05-MAMM-A-333R2.1

547 Pereira J. A., 2010. Activity pattern of Geoffroy's cats (Leopardus geoffroyi) during a

548 period of food shortage. J. Arid Environ. 74, 1106 - 1109.

549 https://doi.org/10.1016/j.jaridenv.2010.03.017

550 Pereira J. A., Di Bitetti M. S., Fracassi N. G., Paviolo A., De Angelo C. D., Di Blanco

551 Y. E., Novaro A. J., 2011. Population density of Geoffroy's cat in scrublands of central

552 Argentina. J. Zool. 283(1), 37 - 44. https://doi.org/10.1111/j.1469-7998.2010.00746.x

553 Pereira J.A., Walker R.S., Novaro A .J. , 2012. Effects of livestock on the feeding and

554 spatial ecology of Geoffroy's cat. J. Arid Environ. 76: 36 - 42.

555 https://doi.org/10.1016/j.jaridenv.2011.08.006 
556 Pereira J., Lucherini M., Trigo T., 2015. Leopardus geoffroyi. The IUCN Red List of

557 Threatened Species 2015: e.T15310A50657011. Available from

558 http://dx.doi.org/10.2305/IUCN.UK.2015-2.RLTS.T15310A50657011.en

559 ( accessed 8 November 2018)

560

561

562

563

564

565

566

567

568

569

570

571

572

573

574

575

576

577

578

579

580

581

582

583

584

585

586

587

588

589

590

591

592

593

594
R Development Core Team, 2015. R: a language and environment for statistical computing. R Foundation for Statistical Computing, Vienna, Austria. Available from http://R-project.org/ (accessed 20 August 2016)

Ridout M. S., Linkie M.,2009. Estimating overlap of daily activity patterns from camera trap data. J. Agr. Biol. Envir. St. 14(3), 322 - 337.

https://doi.org/10.1198/jabes.2009.08038

Robinson T. P., Wint G. W., Conchedda G., Van Boeckel T. P., Ercoli V., Palamara E.,et al., 2014. Mapping the global distribution of livestock. PloS one. 9(5), e96084. https://doi.org/10.1371/journal.pone.0096084

Royle J. A., Chandler R. B., Sollmann R., Gardner B., 2013. Spatial capture-recapture. Academic Press, Waltham.

Rytwinski T., Fahrig L., 2012. Do species life history traits explain population responses to roads? A meta-analysis. Biol. Cons. 147(1), 87 - 98.

https://doi.org/10.1016/j.biocon.2011.11.023

Sikes R.S. 2016.The Animal Care and Use Committee Of The American Society Of Mammalogists. Guidelines of the American Society of Mammalogists for the use of wild mammals in research and education. J. Mammal. 97, $663-688$. https://doi.org/10.1093/jmammal/gyw078

Soler L., Lucherini M., Manfredi C., Ciuccio M., Casanave E. B., 2009. Characteristics of defecation sites of the Geoffroy's cat Leopardus geoffroyi. Mastozool. Neotrop. $16(2), 485-489$.

Sousa K. S., Bager A., 2008. Feeding habits of Geoffroy's cat (Leopardus geoffroyi) in southern Brazil. Mamm. Biol. 73(4), 303 - 308.

https://doi.org/10.1016/j.mambio.2007.04.001

Soutullo Á., Sosa, B. Scarlato G., 2010. Especies prioritarias para la conservación en Uruguay, 2009. Articulación interinstitucional e integración de saberes en la construcción de conocimiento. Agrociencia. 14(3), 114 - 117.

Tirelli F. P., Trigo T. C., Trinca C. S., Albano A. P. N., Mazim F. D., Queirolo D., et al., 2018. Spatial organization and social dynamics of Geoffroy's cat in the Brazilian pampas. J. Mammal. 99(4), 859 - 873. https://doi.org/10.1093/jmammal/gyy064

Trigo,T. C., Tirelli F. P., Machado L. F., Peters F. B., Indrusiak C. B., Mazim F. D., et al., 2013. Geographic distribution and food habits of Leopardus tigrinus and $L$. geoffroyi (Carnivora, Felidae) at their geographic contact zone in southern Brazil. Stud. Neotrop. Fauna Environ. 48(1), 56 - 67. https://doi.org/10.1080/01650521.2013.774789 
595 Walter W. D., Fischer J. W., 2016. Manual of Applied Spatial Ecology. Walter Applied 596 Spatial Ecology Lab, Pennsylvania State University, University Park.. Available from

597 https://ecosystems.psu.edu/research/labs/walter-lab/manual (accessed 06 April 2018).

598

599

600

601

602

603

604

605

606

607

608

609

610

611

612

613

614

615

616

617 
618 Table 1. Capture probability, movement parameter $(\mathrm{km})$, home range size $\left(\mathrm{km}^{2}\right)$ and population density estimation (obtained through Spatially Explicit capture-recapture

620 'SECR' models ) of the best model (bk) assessed for Geoffroy's cats in the Brazilian 621 pampas. CT: camera trap data; TL: radiotelemetry data.

\begin{tabular}{ccccc}
\hline & $\begin{array}{c}\text { Capture } \\
\text { probability } \\
(\mathrm{g} 0 \pm \mathrm{SE})\end{array}$ & $\begin{array}{c}\text { Movement } \\
\text { parameter } \\
(\sigma \pm \mathrm{SE})\end{array}$ & $\begin{array}{c}\text { Home range } \\
\text { size }^{\mathrm{a}} \\
(\text { average } \pm \mathrm{SD})\end{array}$ & $\begin{array}{c}\text { Density } \\
\text { (Individuals per } \\
\left.100 \mathrm{~km}^{2} \pm \mathrm{SE}\right)\end{array}$ \\
\hline $\begin{array}{c}\text { Summer } \\
\text { Winter }\end{array}$ & $0.03 \pm 0.01$ & $0.88 \pm 0.15$ & - & $41.78 \pm 16.12$ \\
$\begin{array}{c}\text { Summer } \\
(\mathrm{CT}+\mathrm{TL})\end{array}$ & $0.01 \pm 0.01$ & $1.05 \pm 0.24$ & - & $41.44 \pm 19.20$ \\
$\begin{array}{c}\text { Winter } \\
(\mathrm{CT}+\mathrm{TL})\end{array}$ & $0.02 \pm 0.01$ & - & $3.53 \pm 3.2$ & $34.54 \pm 13.51$ \\
\hline
\end{tabular}

622

623

624

625

626

627

628

629

630

631

632

633

634

635

636

637

638

639

640

${ }^{\text {a }}$ Estimated by MCP $100 \%$.

.

(1)

(1)

\footnotetext{
${ }^{\mathrm{b}}$ These results were likely biased by telemetry data that included only two females.
} 


\begin{tabular}{|c|c|c|c|c|c|c|}
\hline & Present study & $\begin{array}{c}\text { Nowell and } \\
\text { Jackson } 1996^{\mathrm{d}}\end{array}$ & Cuellar et al. 2006 & \multicolumn{2}{|c|}{ Pereira et al. 2011} & Caruso et al. 2012 \\
\hline Location & Privately owned areas & $\begin{array}{l}\text { Torres Del Paine } \\
\text { National Park }\end{array}$ & $\begin{array}{c}\text { Kaa-Iya del Gran } \\
\text { Chaco National Park }\end{array}$ & $\begin{array}{c}\text { Lihue Calel } \\
\text { National Park }\end{array}$ & Privately owned areas & $\begin{array}{c}\text { Privately owned } \\
\text { areas }\end{array}$ \\
\hline Ecoregion & Uruguayan Savannah & $\begin{array}{c}\text { Magellanic } \\
\text { subpolar forest }\end{array}$ & Dry Chaco & \multicolumn{2}{|c|}{ Low Monte-Espinal } & Espinal \\
\hline Country & Brazil & Chile & Bolivia & \multirow{2}{*}{\multicolumn{2}{|c|}{ Argentina }} & Argentina \\
\hline Human impact ${ }^{\mathrm{a}}$ & medium & low & Low & & & medium \\
\hline $\begin{array}{l}\text { Other felids } \\
\text { recorded }\end{array}$ & Margay, feral cats (rare) & Puma & $\begin{array}{c}\text { Jaguar, puma, } \\
\text { jaguarundi, ocelot, } \\
\text { margay }\end{array}$ & $\begin{array}{l}\text { Pampas cat and } \\
\text { jaguarundi (rare) }\end{array}$ & none & Pampas cat \\
\hline $\begin{array}{l}\text { Suitability } \\
\text { Density estimates }\end{array}$ & $0.25-0.50$ & $0.25-0.50$ & $0.25-0.50$ & \multicolumn{2}{|c|}{$>0.50$} & $>0.50$ \\
\hline $\begin{array}{l}\text { SECR } \\
(\mathrm{CT}+\text { Telemetry })\end{array}$ & $\begin{array}{c}34.54(16.48-72.37) \text { and } \\
63.22(30.14-132.58)^{\mathrm{c}}\end{array}$ & - & - & - & - & - \\
\hline SECR (CT only) & $\begin{array}{c}41.78(20.14-86.70) \text { and } \\
41.44(17.46-98.36)\end{array}$ & - & - & - & - & $45(22.95-88.28)$ \\
\hline Telemetry only & 32.91 & $7-12$ & - & - & - & 50.22 \\
\hline MMDMOSA & $\begin{array}{c}32.44(32.44-36.05) \text { and } \\
43.25(43.25-108.15)\end{array}$ & - & - & - & - & - \\
\hline $\mathrm{HR}$ radius & $\begin{array}{l}51.16(51.16-56.85) \text { and } \\
68.22(68.22-170.553)\end{array}$ & - & - & $140(94-274)$ & $\begin{array}{l}240(189-321) \text { and } \\
230(155-374)\end{array}$ & $\begin{array}{l}21.94(21.94- \\
60.31)\end{array}$ \\
\hline MMDM & $\begin{array}{c}38.05(38.05-42.281) \text { and } \\
50.74(50.74-126.85)\end{array}$ & - & - & $100(66-191)$ & $\begin{array}{c}120(98-167) \text { and } 160 \\
(109-262)\end{array}$ & $16.21(16.21-44.56)$ \\
\hline HMMDM & $\begin{array}{c}87.89(87.89-97.65) \text { and } \\
117.18(117.18-292.97) \\
\end{array}$ & - & $9.52-42.11$ & $190(122-355)$ & $\begin{array}{l}220(170-290) \text { and } \\
290(197-473)\end{array}$ & $24.18(24.18-66.50)$ \\
\hline
\end{tabular}

${ }^{a}$ Definition: low (reserve areas), medium (farmlands with 20-100 cattle/km²[Robinson et al.(2014]), high (farmlands >100 cattle/km²[Robinson et al.(2014]).

${ }^{\mathrm{b}}$ Extracted from Cuyckens et al. (2016);

${ }^{\mathrm{c}}$ In winter we could only include telemetry data from two females which likely biased the combined density estimates.

${ }^{\mathrm{d}}$ W. Johnson, personal communication, in Nowell and Jackson (1996). 
Table 3. Resource selection function analysis using General Linear Models for intrapopulations of radio-tracked Geoffroy's cats in the Brazilian pampas using three different variables: habitat composition, distance to roads and distance to water. $\mathrm{SE}=$ standard error, $\mathrm{LL}=$ lower limit, $\mathrm{UL}=$ upper limit

\begin{tabular}{|c|c|c|c|c|c|c|}
\hline & Estimate & $\mathrm{SE}$ & LL & UL & Z-value & P-value \\
\hline Native forest & 0.35 & 0.16 & 0.04 & 0.67 & 2.23 & $0.02 *$ \\
\hline Grassland & -0.04 & 0.13 & -0.29 & 0.23 & -0.27 & 0.79 \\
\hline Distance to roads & 0 & 0 & 0 & 0 & 3.95 & $7.92 \mathrm{e}-05 * * *$ \\
\hline Distance to water & 0 & 0 & 0 & 0 & -4.98 & $6.26 \mathrm{e}-07 * * *$ \\
\hline
\end{tabular}




\section{Figure Captions}

Fig. 1. Location of the study site in southernmost Brazil, along with a characterization of its habitat composition (NF - Native forest, CR - Cropland, GR - Grassland), camera trap layout (black hexagons) and telemetry relocations (white circles) of radio-collared Geoffroy's cats. Coordinate system: UTM Zone 21J.

Fig. 2. Map of the study site showing Resource Selection Functions (RSF) with prediction classes based on data from telemetry relocations of radio-collared Geoffroy's cats and from variables of habitat composition, distance to roads and distance to water. Coordinate system: UTM Zone 21J.

Fig. 3. Kernel-density estimates of the daily activity patterns of Geoffroy's cat in the Brazilian pampas based on camera trap data. The short vertical lines above the $\mathrm{x}$-axis indicate the times of individual photographs $(n=513)$ and the black solid vertical lines indicate the mean time of sunrise (07:02) and sunset (19:04) across the whole year (Supplementary material S5).

Fig. 4. Kernel-density estimates for activity patterns of Geoffroy's cats in the Brazilian pampas based on camera trap data in summer $(n=240)$ and winter $(n=273)$. The solid and dashed vertical lines indicate the mean time of sunrise and sunset in summer (06:57 and 20:03) and winter (07:15 and 18:10), respectively (Supplementary material S5).

Fig. 5. Circadian activity patterns of Geoffroy's cat in the Brazilian pampas. Bars indicate the proportion of active records with respect to the total number of fixes (in the case of telemetry data) and of records taken (for camera trap data) at that hour of the day. Grey line is the Standard deviation ellipse. (a) Telemetry data ( $n=751$ fixes from 6 individuals). (b) Camera trapping data ( $\mathrm{n}=513$ photos). 


\section{Supplemental materials}

Supplementary material S1. Capture-Recapture methods to estimate density.

Supplementary material S2. Density estimation models ('secr' package [Efford 2018] in R) and their corresponding AIC values for two surveys, including analyses with only camera-trap data (summer and winter) and camera-trap data combined with telemetry data (summer combined and winter combined). Parameters include: predictor variables ${ }^{\mathrm{a}}$, $\mathrm{g} 0=$ baseline detection, sigma $=$ scale parameter. Additionally, $\mathrm{P}=$ Parameters; $\operatorname{logLik}=$ strength of evidence of each model; AICc = AIC with small sample bias adjustment, $\triangle \mathrm{AICc}=$ difference between $\mathrm{AICc}$ model and the best model; $\mathrm{AICcwt}=$ weight attributed to each model.

Supplementary material S3. Estimation of habitat available area for each individual using Minimum Convex Polygon 100\% (MCP) from Tirelli et al. (2018).

Supplementary material S4. General Linear Models ('lme4' package (Bates et al., 2015) in R) and their corresponding AIC values. BIC= Bayesian information criterion; $\operatorname{logLik}=$ strength of evidence of each model; deviance=measure of goodness of fit; df.resid =residual degrees-of-freedom.

Supplementary material S5. Mean and standard deviation for time of sunrise, time of sunset and duration of daylight collected during the entire study period of study and divided between two seasons; data obtained for the Alegrete municipality (which includes most of our study site), RS, Brazil.

Supplementary material S6. Variables used in Resource Selection Function analyses performed in this study. a) Habitat composition (with three categories: native forest, grassland and cropland). b) Distance to road (meters). c) Distance to water (meters). Coordinate system UTM Zone 21J. 
Supplementary material S7. Seasonal activity patterns of Geoffroy's cats in the Brazilian pampas in two different seasons. Bars indicate the proportion of camera trap records taken at that hour of the day. Grey line is the Standard deviation ellipse. A. Spring-summer ( $n=240)$. B. Fall-winter $(n=273)$.

Supplementary material S8. Circadian activity patterns of Geoffroy's cat in the Brazilian pampas in three different habitats: A. native forest, B. croplands, and C. grasslands. Bars indicate the proportion of active records with respect to the total number of fixes at that hour of the day. Arrow represents the mean time of activity. 
Supplementary material S1. Capture-Recapture methods to estimate density

\section{Material and Methods}

Traditional Capture-Recapture methods were used here to compare our results with those reported by other density studies. First, we estimated abundance $(N)$ model results for both survey periods (summer and winter); this was carried out in the software CAPTURE (Rexstad \& Burnham, 1991), and we selected the best model using the model selection criteria (based on a goodness-of-fit test) with same software (Otis, 1978). Second, we calculated the area effectively sampled (AES) applying four different approaches of buffer-width estimation: 1) mean maximum distance moved by individuals outside the area delineated by the outer traps (MMDMOSA); 2) mean maximum distance moved (MMDM); 3) half mean maximum distance moved (HMMDM) (Balme et al., 2009); and 4) the radius of the mean adult home-range size of the studied population (HR radius) (Pereira et al. 2011). The estimations were computed for the Geoffroy's cats living in the area. Third, we calculated density by dividing $N$ of each survey period by the values of $A E S$ generated from with method. Density was also estimated by the telemetry data only; to do so, we assessed the HR of radio-collared individuals with the 95\% kernel approach; home range overlaps were then corrected and the total area occupied by monitored cats was calculated (Caruso et al., 2012). Finally, the total number of individuals monitored was divided by the estimated AES. All AES analyses were calculated using ArcGIS 10.4.1 (ESRI, 2016).

\section{Results}

The best models to explain the capture and recapture of the species were $M(0)=0.95$ and $\mathrm{M}(\mathrm{h})=1.00$ in the summer and $\mathrm{M}(0)=1.00$ and $\mathrm{M}(\mathrm{h})=0.91$ in the winter. White et al. (1982) suggested precaution in the use of the null model, $\mathrm{M}(0)$, especially in a situation that includes a small sample size or possibility of individual heterogeneity. 
Therefore, here we used the $\mathrm{M}(\mathrm{h})$ model, which assumes heterogeneity among individuals in their capture probabilities (White et al. 1982). Our sampling structure was not validated for the closure test provided by CAPTURE; as our surveys were performed in 48 days, we assumed no change in the population during these periods (Pereira et al. 2011; Caruso et al. 2012). The capture probability using CAPTURE was 0.09 for summer and 0.05 for winter, and the abundance was $09( \pm 1.98 \mathrm{SE})$ for summer (CI 7-10) and $12( \pm 3.02 S E)$ for winter (CI 12-30). By using MMDMOSA $(1.73 \mathrm{~km} \pm$ $0.83)$ the $A E S$ was $27.74 \mathrm{~km}^{2}$; with MMDM $(1.52 \mathrm{~km} \pm 0.88 S D)$ the $A E S$ was 23.65 $\mathrm{km}^{2}$; by using HMMDM $(0.76 \mathrm{~km} \pm 0.44 \mathrm{SD})$ it was $10.24 \mathrm{~km}^{2}$; and with HR radius the total sample area was $17.59 \mathrm{~km}^{2}$. Finally, from the seven radio-collared Geoffroy's cat HRs, the total sampled area with telemetry was $21.27 \mathrm{~km}^{2}$. All density estimations were than calculated (Table 2).

\section{References}

Balme, G. A., Hunter, L. T. B, Slotow, R., 2009. Evaluating methods for counting cryptic carnivores. J. Wildlife Manage. 73, 433-441.

Caruso, N., Manfredi, C., Luengos Vidal, E. M., Casanave, E. B., Lucherini, M., 2012. First density estimation of two sympatric small cats, Leopardus colocolo and Leopardus geoffroyi, in a scrubland area of central Argentina. Annal. Zool. Fennici 49, 18-191.

Environmental Systems Research Institute-ESRI 2016. ArcGIS 10.4.1 desktop. Environmental Systems Research Institute, Redlands, CA.

Otis, D. L., Burnham, K. P., White, G. C., Anderson, D.R,. 1978. Statistical inference from capture data on closed populations. Wildl. Monogr. 62, 1-135.

Pereira, J. A., Di Bitetti, M. S., Fracassi, N. G., Paviolo, A., De Angelo, C. D., Di

Blanco, Y. E., Novaro, A. J., 2011. Population density of Geoffroy's cat in scrublands of central Argentina. J. Zool. 283(1), 37-44.

Rexstad, D. L., Burnham, K. P., 1991. User's guide for interactive program CAPTURE. Abundance estimation of closed animal populations. Fort Collins: Colorado State University.

White, G. C., D. R. Anderson, K. P. Burnham, D. L. Otis. 1982. Capture-recapture and removal methods for sampling closed populations. Los Alamos National Laboratory Publication LA-8787-NERP. Los Alamos, New Mexico, USA. 
Supplementary material S2. Density estimation models ('secr' package (Efford 2018) in $\mathrm{R}$ ) and their corresponding AIC values to two surveys for analyses using only camera trap data (summer and winter) and combined with telemetry data (summer combined and winter combined). Parameters include: predictor variables ${ }^{\mathrm{a}}, \mathrm{g} 0=$ baseline detection, sigma $=$ scale parameter. Additionally, $\mathrm{P}=$ Parameters; $\operatorname{logLik}=$ strength of evidence of each model; $\mathrm{AIC}_{\mathrm{c}}=\mathrm{AIC}$ with small sample bias adjustment, $\Delta \mathrm{AIC}_{\mathrm{c}}=$ difference between $\mathrm{AIC}_{\mathrm{c}}$ model and the best model; $\mathrm{AIC}_{\mathrm{cwt}}=$ weight attributed to each model.

\begin{tabular}{|c|c|c|c|c|c|c|c|}
\hline \multicolumn{2}{|c|}{$\begin{array}{c}\text { Model } \\
\text { (detection function= half } \\
\text { normal) }\end{array}$} & $\mathrm{P}$ & $\operatorname{logLik}$ & AIC & $\mathrm{AIC}_{\mathrm{c}}$ & $\Delta \mathrm{AIC}_{\mathrm{c}}$ & $\mathrm{AIC}_{\mathrm{cwt}}$ \\
\hline \multicolumn{8}{|c|}{ Summer } \\
\hline bk & g0 bk sigma 1 & 3 & -231 & 469 & 472.021 & 0 & 0.75 \\
\hline $\mathrm{Bk}$ & g0 Bk sigma 1 & 3 & -233 & 472 & 475.294 & 3.273 & 0.15 \\
\hline sex & $\mathrm{g} 0 \sim 1$ sigma 1 & 2 & -236 & 476 & 477.411 & 5.39 & 0.05 \\
\hline 0 & $\mathrm{~g} 0 \sim \mathrm{b}$ sigma $\sim 1$ & 3 & -235 & 476 & 479.468 & 7.447 & 0.02 \\
\hline $\mathrm{h}$ & g0 Sex sigma 1 & 3 & -236 & 478 & 480.944 & 8.923 & 0.01 \\
\hline $\mathrm{b}$ & $\mathrm{g} 0 \sim \mathrm{h} 2$ sigma $\sim 1 \mathrm{pmix} \sim \mathrm{h} 2$ & 4 & -236 & 480 & 486.578 & 14.56 & 0 \\
\hline \multicolumn{8}{|c|}{ Winter } \\
\hline bk & g0 bk sigma 1 & 3 & -152 & 309 & 313.204 & 0 & 0.85 \\
\hline $\mathrm{Bk}$ & g0 Bk sigma 1 & 3 & -154 & 313 & 317.222 & 4.018 & 0.11 \\
\hline sex & $\mathrm{g} 0 \sim 1$ sigma $\sim 1$ & 2 & -157 & 318 & 319.777 & 6.573 & 0.03 \\
\hline 0 & $\mathrm{~g} 0 \sim \mathrm{b}$ sigma 1 & 3 & -157 & 320 & 323.915 & 10.71 & 0 \\
\hline $\mathrm{h}$ & g0 Sex sigma $\sim 1$ & 3 & -157 & 320 & 324.059 & 10.86 & 0 \\
\hline $\mathrm{b}$ & $\mathrm{g} 0 \sim \mathrm{h} 2$ sigma $\sim 1 \mathrm{pmix} \sim \mathrm{h} 2$ & 4 & -157 & 322 & 330.063 & 16.86 & 0 \\
\hline \multicolumn{8}{|c|}{ Summer combined } \\
\hline bk & lambda0 bk sigma 1 hazard & 3 & -541 & 1089 & 1092.21 & 0 & 1 \\
\hline $\mathrm{Bk}$ & lambda0 Bk sigma 1 hazard & 3 & -549 & 1104 & 1107.3 & 15.09 & 0 \\
\hline sex & lambda0 1 sigma 1 hazard & 2 & -553 & 1111 & 1112.09 & 19.88 & 0 \\
\hline 0 & lambda0 b sigma 1 hazard & 3 & -551 & 1109 & 1112.12 & 19.91 & 0 \\
\hline $\mathrm{h}$ & lambda0 Sex sigma 1 hazard & 3 & -552 & 1110 & 1113.12 & 20.91 & 0 \\
\hline $\mathrm{b}$ & $\begin{array}{l}\text { lambda0 h2 sigma 1 } \\
\text { pmix } \sim \text { h2 hazard }\end{array}$ & 4 & -550 & 1109 & 1115.41 & 23.2 & 0 \\
\hline \multicolumn{8}{|c|}{ Winter combined } \\
\hline bk & lambda0 bk sigma 1 hazard & 3 & -230 & 465 & 469.184 & 0 & 0.43 \\
\hline $\mathrm{Bk}$ & lambda0 Bk sigma 1 hazard & 3 & -230 & 466 & 470.104 & 0.92 & 0.27 \\
\hline sex & lambda0 1 sigma 1hazard & 2 & -233 & 470 & 471.285 & 2.101 & 0.15 \\
\hline 0 & lambda0 bsigma 1hazard & 3 & -233 & 472 & 475.569 & 6.385 & 0.02 \\
\hline $\mathrm{h}$ & lambda0 Sexsigma 1hazard & 3 & -232 & 470 & 473.934 & 4.75 & 0.04 \\
\hline $\mathrm{b}$ & $\begin{array}{c}\text { lambda0 } \sim \mathrm{h} 2 \\
\text { sigma } 1 \mathrm{pmix} \sim \mathrm{h} 2 \mathrm{hazard}\end{array}$ & 4 & -228 & 464 & 472.325 & 3.141 & 0.09 \\
\hline
\end{tabular}

a Predictor variables used in the construction of density models in "secr" package (Efford 2018): $\mathrm{bk}=$ Animal $\mathrm{x}$ site learned response; $\mathrm{Bk}=$ Animal $\mathrm{x}$ site transient response; sex= Capture probabilities differ between sexes; $0=$ Capture probabilities are homogeneous among individuals; $\mathrm{h}=$ Individual heterogeneity; and $b=$ Learned response. 
Supplementary material S3. Estimation of habitat available for each individual using Minimum Convex Polygon 100\% (MCP) from Tirelli et al. (2018).

\begin{tabular}{ccc}
\hline Individual & N relocations & $100 \% \mathrm{MCP}\left(\mathrm{km}^{2}\right)$ \\
\hline M1 & 59 & 2.46 \\
M2 & 54 & 1.87 \\
M3 & 23 & 0.86. \\
M4 & 62 & 6.18 \\
F1 & 34 & 0.75 \\
F2 & 133 & 1.87 \\
F3 & 69 & 0.63 \\
\hline
\end{tabular}


Supplementary material S4. General Linear Models ('lme4' package (Bates et al., 2015 ) in R) and their corresponding AIC values. BIC = Bayesian information criterion; $\log$ Lik $=$ strength of evidence for each model; deviance=measure of goodness of fit; df.resid =residual degrees-of-freedom.

\begin{tabular}{lccccc}
\hline \multicolumn{1}{c}{ Models } & AIC & BIC & logLik & deviance & df.resid \\
\hline use relevel(veg,"CR")+ d_road + & 2573.0 & 2610.7 & -1280.5 & 2561.0 & 3928 \\
d_river+(1|id) & 2591.3 & 2610.1 & -1292.6 & 2585.3 & 3931 \\
use d_river+(1|id) & 2592.8 & 2624.2 & -1291.4 & 2582.8 & 3929 \\
use veg + d_road+(1|id) & 2614.9 & 2633.7 & -1304.4 & 2608.9 & 3931 \\
use d_road +(1|id) & 2625.4 & 2650.5 & -1308.7 & 2617.4 & 3930 \\
use relevel(veg,"RV")+(1|id) & 2625.4 & 2650.5 & -1308.7 & 2617.4 & 3930 \\
use relevel(veg,"GR")+(1|id) & 2625.4 & 2650.5 & -1308.7 & 2617.4 & 3930 \\
use relevel(veg,"CR")+(1|id) & 2625.4 & 2650.5 & -1308.7 & 2617.4 & 3930 \\
use veg+(1|id) & 2663.0 & 2675.5 & -1329.5 & 2659.0 & 3932 \\
use 1|id & & & & & \\
\hline
\end{tabular}


Supplementary material S5. Mean (hours:minutes) and standard deviation (minutes) for time of sunrise, time of sunset and duration of daylight collected during the entire study period of study and divided between two seasons; data obtained for the Alegrete municipality (which includes most of our study site), RS, Brazil.

\begin{tabular}{lcccccc}
\hline & \multicolumn{2}{c}{ Sunrise } & \multicolumn{2}{c}{ Sunset } & \multicolumn{2}{c}{ Duration of daylight } \\
& Mean & SD & Mean & SD & Mean & SD \\
\hline All seasons & $07: 02$ & 0.98 & $19: 04$ & 2.72 & $12: 01$ & 3.35 \\
Summer-spring & $06: 57$ & 0.69 & $20: 03$ & 1.83 & $13: 16$ & 3.25 \\
Fall-winter & $07: 15$ & 0.84 & $18: 10$ & 0.69 & $10: 54$ & 1.50 \\
\hline
\end{tabular}

*Data base collected from (C) 2011-2016 sunrise-and-sunset.com 

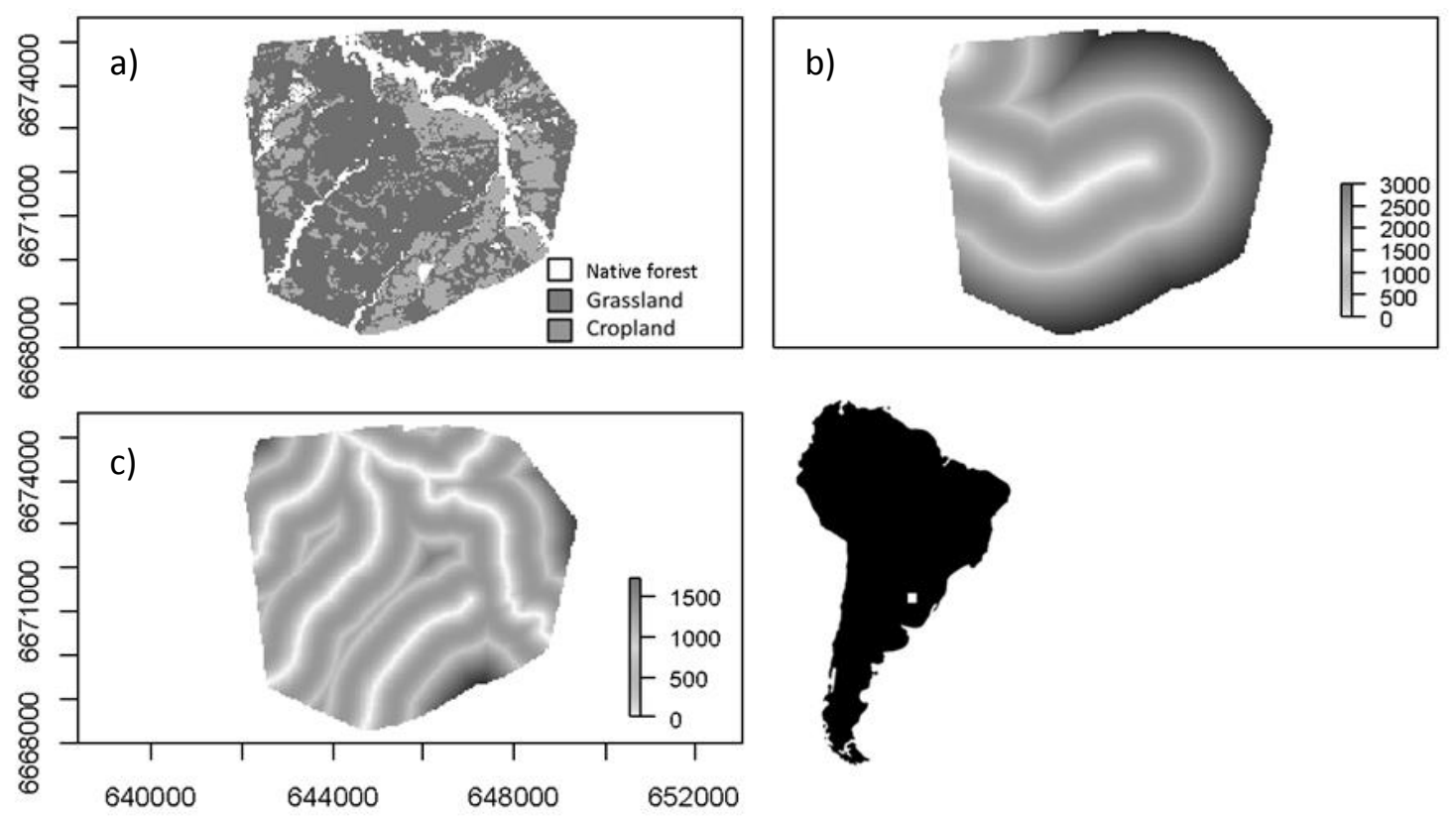

Supplementary material S6. Variables used in Resource Selection Function analyses performed in this study. a) Habitat composition (with three categories: native forest, grassland and cropland). b) Distance to road (meters). c) Distance to water (meters). Coordinate system UTM Zone 21J. 

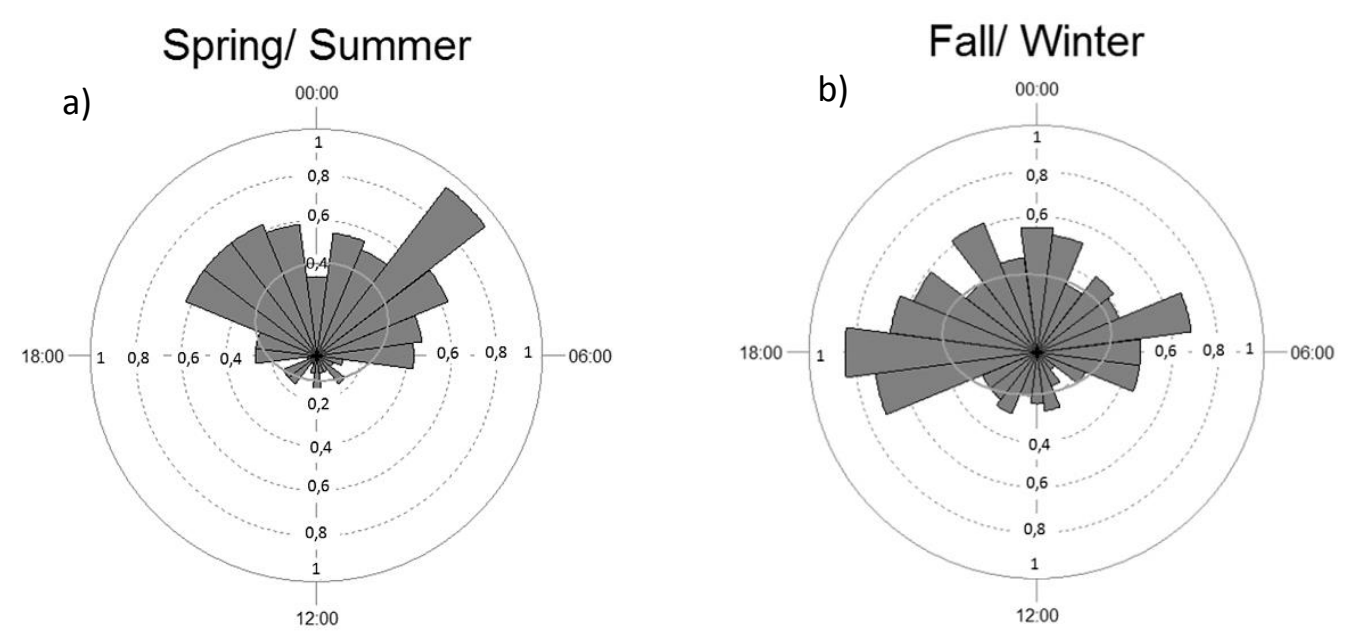

Supplementary material S7. Seasonal activity patterns of Geoffroy's cats in the Brazilian pampas in two different seasons. Bars indicate the proportion of camera-trap records taken at that hour of the day. Grey line is the Standard deviation ellipse. a) Spring-summer ( $n=240)$. b) Fall-winter $(n=273)$. 
a)
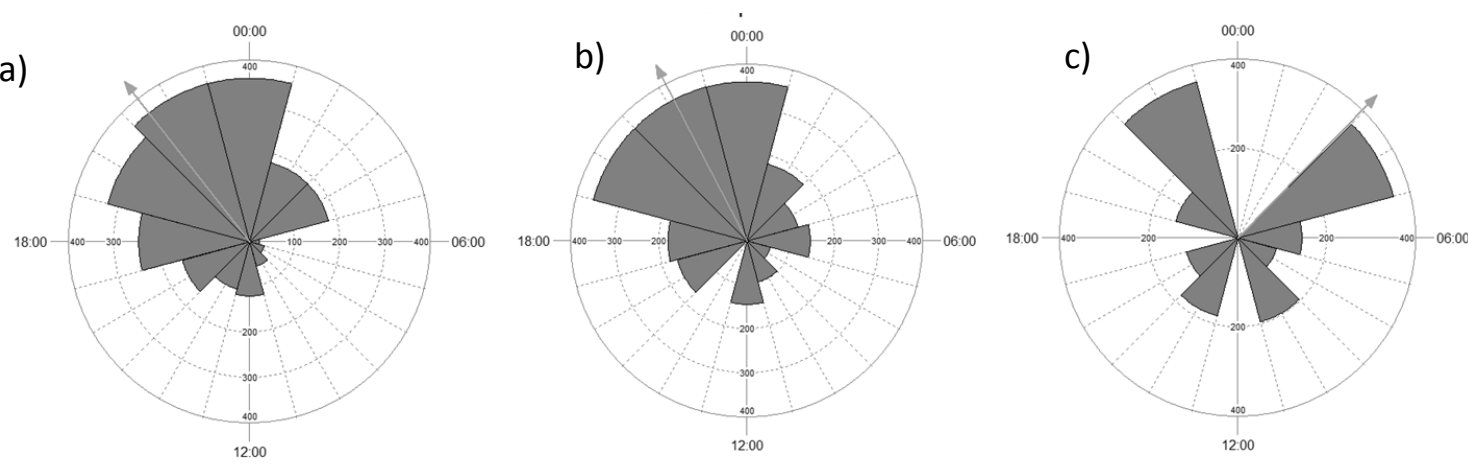

Supplementary material S8. Circadian activity patterns of Geoffroy's cats in the Brazilian pampas in three different habitats: a) native forest, b) croplands, and c) grasslands. Bars indicate the proportion of active records with respect to the total number of fixes at that hour of the day. Arrows represent the mean time of activity. 